\title{
Complex inner core boundary from frequency characteristics of the reflection coefficients of PKiKP waves observed by Hi-net
}

\author{
Satoru Tanaka ${ }^{1 *}$ and Hrvoje Tkalčić ${ }^{2}$
}

\begin{abstract}
Frequency-dependent reflection coefficients of $\mathrm{P}$ waves at the inner core boundary (ICB) are estimated from the spectral ratios of PKiKP and PCP waves observed by the high-sensitivity seismograph network (Hi-net) in Japan. The corresponding PKiKP reflection locations at the ICB are distributed beneath the western Pacific. At frequencies where noise levels are sufficiently low, spectra of reflection coefficients show four distinct sets of characteristics: a flat spectrum, a spectrum with a significant spectral hole at approximately 1 or $3 \mathrm{~Hz}$, a spectrum with a strong peak at approximately 2 or $3 \mathrm{~Hz}$, and a spectrum containing both a sharp peak and a significant hole. The variety in observed spectra suggests complex lateral variations in ICB properties. To explain the measured differences in frequency characteristics of ICB reflection coefficients, we conduct 2D finite difference simulations of seismic wavefields near the ICB. The models tested in our simulations include a liquid layer and a solid layer above the ICB, as well as sinusoidal and spike-shaped ICB topography with varying heights and scale lengths. We find that the existence of a layer above the ICB can be excluded as a possible explanation for the observed spectra. Furthermore, we find that an ICB topographic model with wavelengths and heights of several kilometers is too extreme to explain our measurements. However, restricting the ICB topography to wavelengths and heights of $1.0-1.5 \mathrm{~km}$ can explain the observed frequency-related phenomena. The existence of laterally varying topography may be a sign of lateral variations in inner core solidification.
\end{abstract}

Keywords: Inner core boundary; Topography; PKiKP; Finite difference modeling

\section{Background}

The inner core boundary (ICB) is one of the vital regions for understanding the Earth's core dynamics (Loper and Roberts 1981; Loper 1983; Bergman and Fearn 1994; Shimizu et al. 2005; Deguen et al. 2007; Sumita and Bergman 2009; Deguen 2012). Seismological studies of the ICB and its inferred characteristics, such as the density jump between the inner and the outer cores, the shear-wave velocity at the top of the inner core, and scattering of seismic energy from small-scale topography at the inner core surface, are important in elucidating the growth mechanism of the inner core and the source of the geodynamo (Souriau 2007; Sumita and Bergman 2009; Deuss 2014; Tkalčić 2015).

\footnotetext{
* Correspondence: stan@jamstec.go.jp

'Department of Deep Earth Structure and Dynamics Research, Japan Agency for Marine-Earth Science and Technology, Yokosuka 237-0061, Japan Full list of author information is available at the end of the article
}

The hypothesis of a hemispherical structure in the upper inner core (Tanaka and Hamaguchi 1997) has been widely accepted because it is supported by seismic observations of body waves and free oscillations (Creager 1999; Deuss et al. 2010). Hemispherical dichotomy is recognized as a global phenomenon near the ICB (Niu and Wen 2001; Waszek et al. 2011) and possibly near the center of the inner core (Lythgoe et al. 2014). To explain the hemispherical structure of the inner core, two models have been proposed: a large-scale asymmetric flow in the outer core (Sumita and Olson 1999; Aubert et al. 2008; Gubbins et al. 2011) and translational convection in the inner core (Alboussiere et al. 2010; Monnereau et al. 2010). The nature of these models is that they allow diametrically opposite scenarios of freezing and melting, i.e., one of the two hemispheres solidifies faster than the other. This has raised further questions about whether the surface of the inner core in 


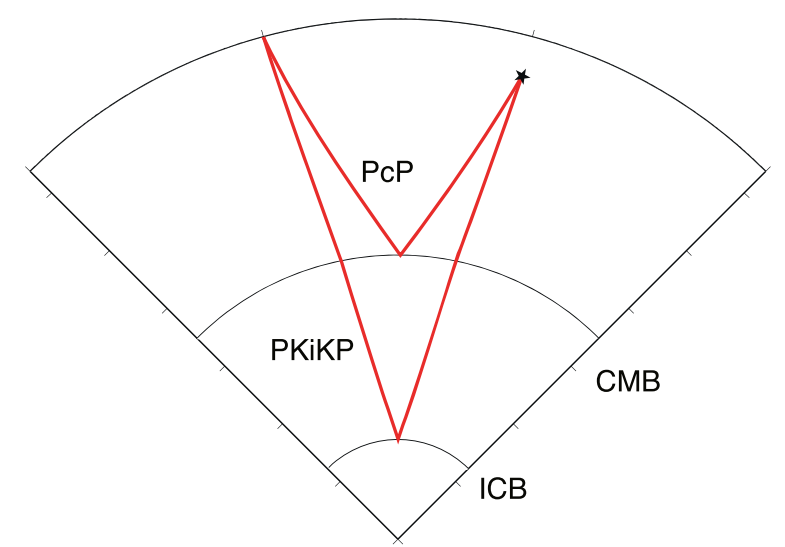

Fig. 1 Ray paths of PCP and PKiKP seismic phases. A cross section of Earth with ray paths of PCP and PKiKP seismic phases originating at the source (star) and recorded by a station at the Earth's surface

the eastern hemisphere is melting or freezing. Although translational convection models predict that the eastern hemisphere is melting, outer-core large asymmetric flow models disagree about which hemisphere is melting, due to the problem setting, e.g., heat flux at the core-mantle boundary $(\mathrm{CMB})$ and outer core dynamics.

Measurements of $\mathrm{PKiKP} / \mathrm{PcP}$ amplitude ratios have been used to infer the density jump at the ICB, as well as to determine the shear velocity at the top of the inner core. A sketch of their ray paths is shown in Fig. 1. Studies in the twentieth century usually analyzed PKiKP recorded on short-period seismographs with a predominant frequency of $1 \mathrm{~Hz}$ (Engdahl et al. 1970; Buchbinder 1972; Engdahl et al. 1974; Souriau and Souriau 1989; Shearer and Masters 1990). These pioneering studies may have been hampered by the small number of observations and the large scatter in PKiKP/ PcP amplitude ratios due to the nature of noisy amplitude measurements (Tkalčić et al. 2009). Another factor influencing the amplitude ratios, and possibly the anticorrelation between the two phases, is the occurrence of near-receiver crustal and mantle heterogeneity (Tkalčić et al. 2010).

The new era of modern instruments, dense networks, and improved global coverage enables observation of a large number of PKiKP and PcP phases on the same seismogram. For example, Koper et al. (2003) analyzed a significant number of PKiKP phases at shorter distances recorded by small aperture arrays of the International Monitoring System. This study was followed by a large number of new studies (Cao and Romanowicz 2004; Koper et al. 2004; Koper and Pyle 2004; Poupinet and Kennett 2004; Koper and Dombrovskaya 2005; Krasnoshchekov et al. 2005; Leyton et al. 2005; Kawakatsu 2006; Leyton and Koper 2007a, b; Peng et al. 2008).
More specifically, Koper and Pyle (2004) measured $\mathrm{PKiKP} / \mathrm{PcP}$ amplitude ratios from seismograms filtered between 1 and $3 \mathrm{~Hz}$. Their analysis did not reveal any differences between the eastern and western hemispheres of the inner core. Using phases from a Mariana event observed in Japan, whose raypaths sampled the ICB beneath the western Pacific, Kawakatsu (2006) found little scattering energy in the PKiKP coda. However, Leyton and Koper (2007b) analyzed the coda of PKiKP and suggested the existence of small-scale heterogeneities in the uppermost inner core. This result indicates that the strong scattering region is located beneath the Pacific Ocean and Asia, which covers parts of the eastern and western hemispheres. On the basis of scattering properties and Q structure, Cormier (2007) inferred textural differences between the eastern and western hemispheres near the surface of the inner core, including vertically oriented structures in the eastern hemisphere.

Interestingly, the existence of a high-frequency PKiKP phase (up to $5 \mathrm{~Hz}$ ) with steep incidence angles at the ICB was first observed by Poupinet and Kennett (2004) using phases recorded by narrow-aperture arrays and temporal broadband networks on the Australian continent, whose reflection points were in the eastern hemisphere. Recently, the same class of PKiKP waves was observed in the eastern hemisphere on Chinese and Japanese short-period and broadband stations (J-array) (Tkalčić et al., 2009, 2010) and by the high-sensitivity seismograph network (Hi-net) in Japan (Dai et al. 2012, Jiang and Zhao 2012).

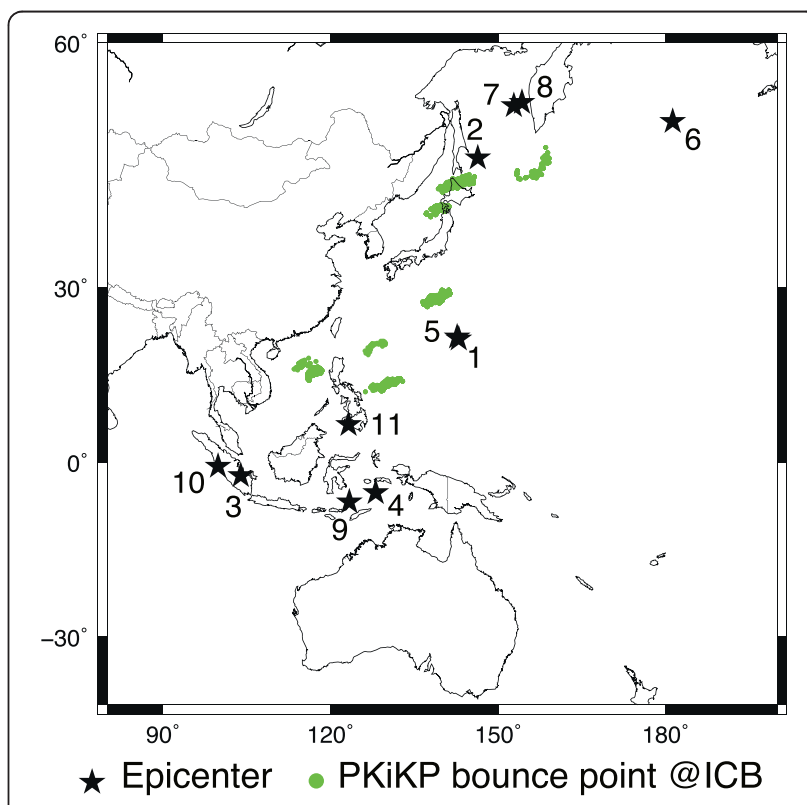

Fig. 2 Study area. Map view of epicenters (stars) and PKiKP bounce points at the ICB (green circles). Event numbers are taken from Table 1 
Table 1 Event list

\begin{tabular}{lllllllll}
\hline Event & Date & Time $($ UTC $)$ & Lat. $\left(^{\circ}\right)$ & Lon. $\left(^{\circ}\right)$ & Depth $(\mathrm{km})$ & $\mathrm{m}_{\mathrm{b}}$ & Remarks & No. of used records \\
\hline 1 & 20010703 & $13: 10: 42.60$ & 21.641 & 142.984 & 290.0 & 6.0 & DC & 73 \\
2 & 20021117 & $04: 53: 48.46$ & 47.946 & 146.419 & 470.2 & 5.8 & Sm & 58 \\
3 & 20040725 & $14: 35: 19.06$ & -2.427 & 103.981 & 582.1 & 6.8 & DC & 83 \\
4 & 20060127 & $16: 58: 53.67$ & -5.473 & 128.131 & 397.0 & 7.0 & Sm & 77 \\
5 & 20070928 & $13: 38: 57.88$ & 22.013 & 142.668 & 260.0 & 6.7 & Sm & 41 \\
6 & 20080322 & $21: 24: 11.27$ & 52.176 & -178.716 & 132.0 & 5.8 & Sm & 65 \\
7 & 20080705 & $02: 12: 04.48$ & 53.882 & 152.886 & 632.8 & 6.8 & DC & 49 \\
8 & 20081124 & $09: 02: 58.76$ & 54.203 & 154.322 & 492.3 & 6.5 & DC & 83 \\
9 & 20090828 & $01: 51: 20.40$ & -7.146 & 123.427 & 642.4 & 6.3 & DC & 31 \\
10 & 20090930 & $10: 16: 09.25$ & -0.720 & 99.867 & 81.0 & 7.1 & DC & 33 \\
11 & 20100723 & $23: 15: 10.19$ & 6.780 & 123.260 & 640.0 & 7.4 & Sm & 53 \\
\hline
\end{tabular}

Hypocenters are taken from the Earthquake data report provided by U.S.G.S.

$D C$ double couple, $\mathrm{Sm}$ smoothed radiation pattern

To contribute to a better understanding of inner core dynamics and to constrain ICB structure in the eastern hemisphere, here, we collect an extensive dataset of PKiKP waves recorded by $\mathrm{Hi}$-net in Japan. Our aim is to shift focus from analyzing a single value of the PKiKP/ PcP amplitude ratio to evaluating its broad frequency characteristics, which is philosophically similar to how Cummins and Johnson (1988) evaluated pre-critical PKiKP waveforms and spectra by using a hybrid full wave-reflectivity algorithm. A dense configuration of borehole seismograms with high SNR observations of PKiKP waves over an unusually broad range of frequencies facilitates this new approach to estimate ICB properties. Thus, we examine data in the frequency domain and investigate possible broader implications for Earth's core dynamics.

\section{Methods}

Hi-net comprises approximately 700 short-period seismographs placed at the bottoms of individual boreholes (Okada et al. 2004). However, even for large-volume datasets such as Hi-net, good records of PKiKP and PcP are not frequently observed. We have detected PcP and PKiKP waveforms from 11 earthquakes with body wave magnitude $\geq 5.8$ and focal depths of $\geq 80 \mathrm{~km}$ around Japan before the 2011 Tohoku earthquake (Fig. 2, Table 1). This covers epicentral distances from $15^{\circ}$ to $50^{\circ}$. The reflection points of PKiKP at the ICB are distributed beneath the western Pacific, which is part of the eastern hemisphere (Fig. 2).

After visual examination of waveforms, amplitude spectra of PKIKP phases, and corresponding waveforms and spectra of pre-arrival noise, we applied a zero-phase Butterworth band-pass filter with corner frequencies of 2 and $5 \mathrm{~Hz}$ to $60 \mathrm{~s}$ record segments centered around theoretical PKiKP arrival times (Fig. 3a, b). This was done for all records except the Mariana event (event 1), which was examined by Kawakatsu (2006) and observed to have large PKiKP signals around $1 \mathrm{~Hz}$. Note that we use band-pass filtering only for initial identification of PKiKP.

After applying the band-pass filter described above and retrieving record segments of $\pm 10 \mathrm{~s}$ length around PKiKP arrivals, seismograms were divided into subgroups comprising 70-230 stations with traces sorted by increasing epicentral distance. We then selected subsets of coherent waveforms by using the cross-correlation matrix method (Tkalčić et al. 2011), which retained 46220 records per event. To find coherent PKiKP arrivals in each sub-group, we empirically determined the minimum percentage $(\tau)$ of all waveform pairs that should cross-correlate in such a way that the average crosscorrelation coefficient equals or exceeds a threshold $\beta$. The algorithm calculates the cross-correlation coefficients for each pair of waveforms and counts the total percentage of pairs with cross-correlation coefficient exceeding $\beta$. For example, for $\beta>0.4, \tau>10 \%$, and 130 total waveforms, we found that 77 waveforms satisfied these criteria and were consequently selected as "mutually coherent" (Fig. 3c). Incoherent waveforms were not used in further analyses. Subsequently, we visually checked the waveforms that satisfied the above criteria to find possible PKiKP signals with high signal-to-noise ratios, which resulted in a station list of "good" sites for PKiKP observations.

In the next step, we prepared a spectrogram of $90 \mathrm{~s}$ length from the unfiltered seismograms, with a sampling interval of $1 \mathrm{~s}$ and a lapse time of $50 \mathrm{~s}$ from the theoretical arrival times of PcP or PKiKP (Fig. 4). Each Fourier spectrum is calculated for a Welch tapered $10 \mathrm{~s}$ window (Press et al. 1988), then smoothed using a three-point moving average. Using the station list obtained with the 


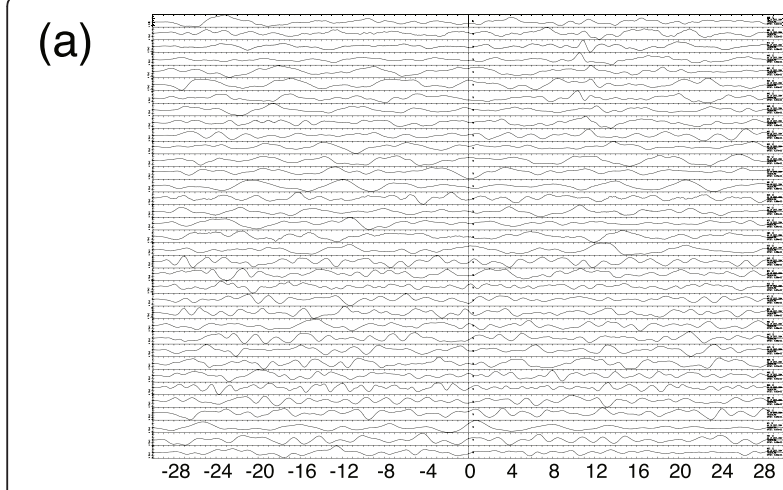

(b)

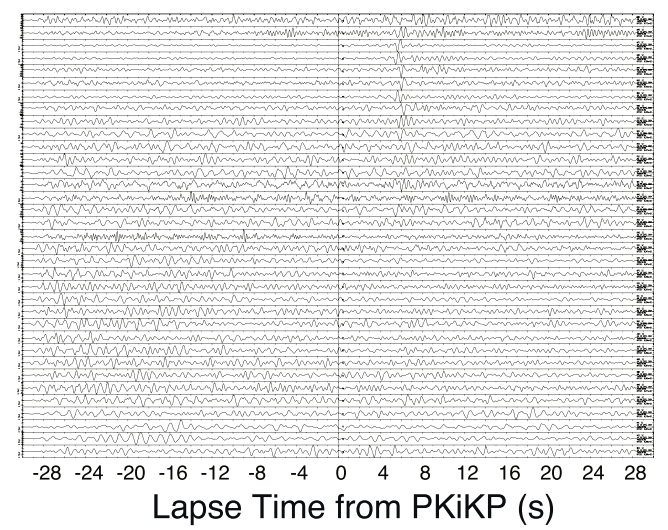

(c) Total 77 BPF waveforms PKiKP (C) $\operatorname{cc}>0.4$ with at least $10 \%$ out of 130 waveforms

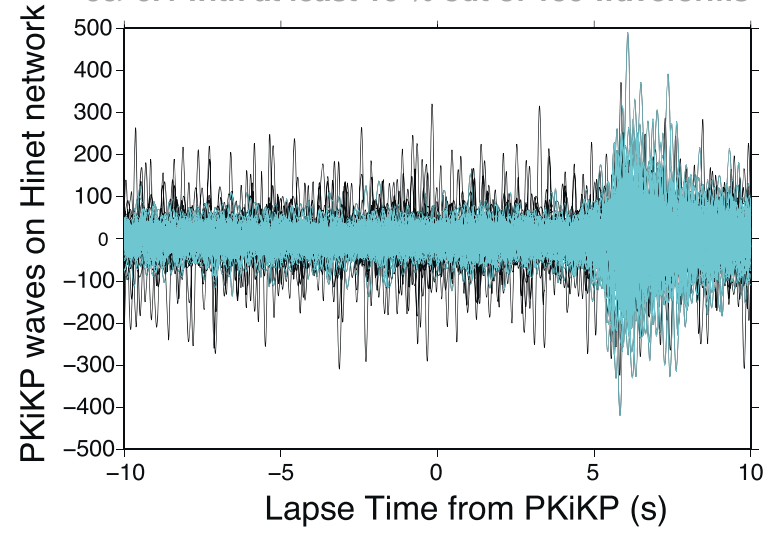

Fig. 3 Selection of coherent waveforms. a Raw seismograms of event 3 recorded by Hi-net stations whose names start with letter "I." Horizontal axis is the lapse time from the theoretical arrival times of PKiKP. $\mathbf{b}$ Seismograms from $\mathbf{a}$ are band-pass filtered with cutoff frequencies of 2 and $5 \mathrm{~Hz}$. c The selected set of coherent waveforms after employing the cross-correlation matrix method (Tkalčić et al., 2011) is shown by cyan lines

cross-correlation matrix method, we used both the seismograms and spectrograms of PKiKP and PcP to identify acceptable data for calculating spectral ratios of PKiKP/PcP (Fig. 5). For each event, between 33 and 83 station records were retained (Table 1). Finally, the mean $\mathrm{PKiKP} / \mathrm{PcP}$ spectral ratio was computed at each frequency that exceeded the mean of the noise/ $\mathrm{PcP}$ ratio (Fig. 5). The spectra of PcP and PKiKP contained several peaks and holes whose central frequencies were not uniform for all events. For example, for event 3, the spectral ratio of $\mathrm{PKiKP} / \mathrm{PcP}$ exceeded the mean noise $/ \mathrm{PcP}$ ratio in the range 0.8 to $3.0 \mathrm{~Hz}$ (Fig. $5 \mathrm{a}$ ). In the case of other events, however, valid frequency ranges were somewhat different, e.g., 2-3 Hz for event 6 (Fig. 5b) and 1-4 Hz for event 11 (Fig. 5c). Despite the above restrictions, the relatively high-frequency content of the PcP and PKiKP waves means that these high-quality simultaneous observations over a broad frequency interval are unprecedented.

To correct the amplitude ratios, we determined focal mechanisms using the program of Kikuchi and Kanamori (2003), rather than cataloged Global CMT solutions, USGS Moment tensor, and double-couple solutions. The broadband displacements of $\mathrm{P}$ and $\mathrm{SH}$ waves in the frequency range $0.002-1 \mathrm{~Hz}$ were used for the inversion. Furthermore, we compared the short-period (SP) $\mathrm{P}$ wave amplitudes $(1-5 \mathrm{~Hz})$ with the radiation pattern predicted from each focal mechanism solution. Although the observed SP amplitudes showed a high degree of scatter, we found that some events had clear energy even near the nodal plane and null axis, suggesting a smoothed radiation pattern. This may be due to scattering near the source region, as discussed in previous studies that determined magnitudes from SP data (e.g., (Schweitzer and Kværna 1999; Takemura et al. 2015). These results are summarized in the Remarks column of Table 1 . The smoothed radiation pattern for the short period is a likely explanation for the observation of PcP and PKiKP in the events from Kuril (event 2) and Mindanao (event 11), in which the take-off azimuths and angles of PcP and PKiKP were located near the nodal plane.

Attenuation factors for the mantle and core at arbitrary frequencies were normalized to a reference frequency of $1 \mathrm{~Hz}$. We then obtained the corrected relationship between spectral and theoretical amplitude ratios from the following relationship:

$$
\begin{aligned}
\left(\frac{A_{\mathrm{PKiKP}}}{A_{\mathrm{PcP}}}\right)_{\text {corrected }} & =\left(\frac{A_{\mathrm{PKiKP}}}{A_{\mathrm{PcP}}}\right)_{\text {observed }} \frac{F_{\mathrm{PcP}}}{F_{\mathrm{PKiKP}}} \frac{\exp \left(-\pi f_{0} \Delta t^{*}\right)}{\exp \left(-\pi f \Delta t^{*}\right)} \\
& =\frac{T_{\mathrm{PK}} R_{\mathrm{KK}} T_{\mathrm{KP}}}{R_{\mathrm{PP}}} \frac{G_{\mathrm{PKiKP}}}{G_{\mathrm{PcP}}} \frac{\exp \left(-\pi f_{0} t_{\mathrm{PKiKP}}^{*}\right)}{\exp \left(-\pi f_{0} t_{\mathrm{PcP}}^{*}\right)},
\end{aligned}
$$

where the subscripts PKiKP and PcP denote the corresponding seismic phases. On the left side of the equation, $A$ is the spectral amplitude at an arbitrary frequency, $F$ is the focal mechanism radiation pattern, $t^{*}$ is the anelastic parameter (Lay and Wallace 1995), and $\Delta t *$ is the differential anelastic parameter between PKiKP 

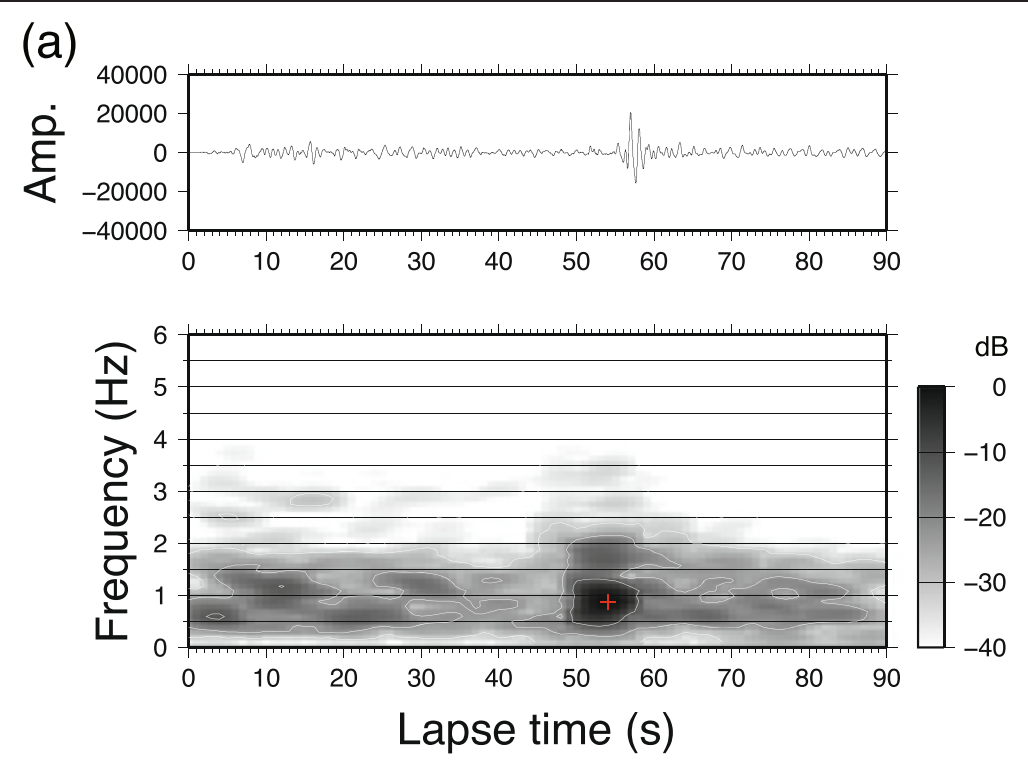

(b)
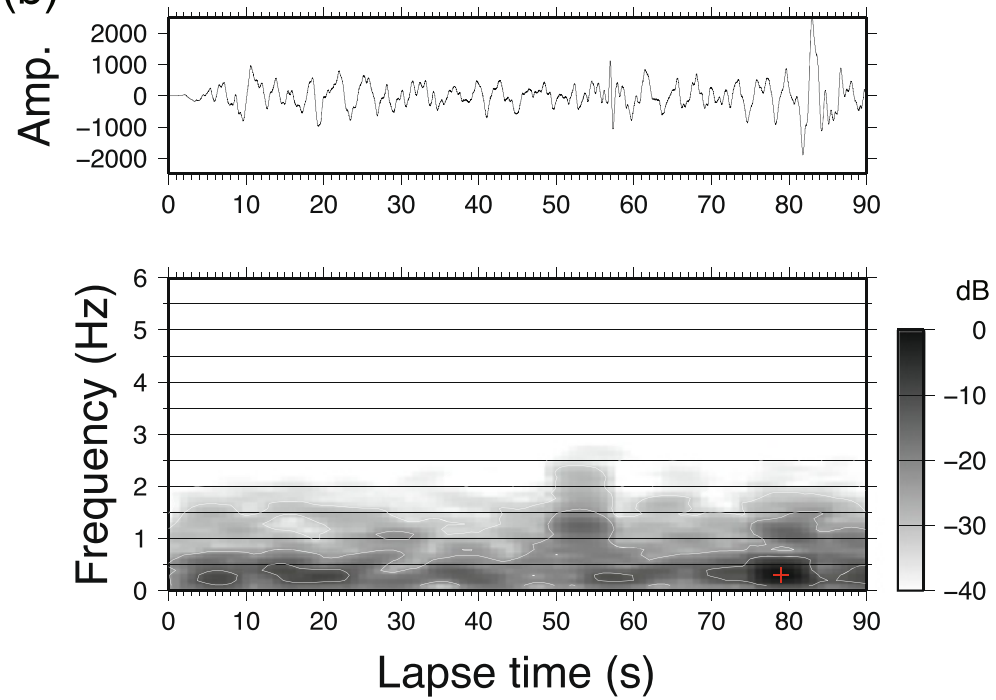

Fig. 4 Spectrograms including PCP and PKiKP. Raw seismograms and spectrograms of event 3 for a PCP and $\mathbf{b}$ PKiKP recorded at station IKTH. Spectrogram scaling is in decibels $(\mathrm{dB})$. The predicted arrival times of PcP and PKiKP are located at a lapse time of $50 \mathrm{~s}$

and PcP. On the right side, $G$ represents the geometrical spreading factor; $f_{0}(=1 \mathrm{~Hz})$ and $f$ are reference and observed frequencies, respectively; $T_{\mathrm{PK}}$ and $T_{\mathrm{KP}}$ are the transmission coefficients at the $\mathrm{CMB}$; and $R_{\mathrm{PP}}$ and $R_{\mathrm{KK}}$ denote reflection coefficients at the CMB and ICB, respectively. The reflection coefficient $R_{\mathrm{KK}}$ at the ICB is estimated from the corrected spectral ratios by considering the terms of the reflection and transmission coefficients at the $\mathrm{CMB}$, the geometrical spreading factor, and anelastic parameters, which are calculated using ak135 (Kennett et al. 1995):
$\left(R_{\mathrm{KK}}\right)_{\text {estimated }}=\left[\left(\frac{A_{\mathrm{PK} \text { iKP }}}{A_{\mathrm{PCP}}}\right)_{\text {corrected }}\right] /\left[\frac{T_{\mathrm{PK}} T_{\mathrm{KP}}}{R_{\mathrm{PP}}} \frac{G_{\mathrm{PKiKP}}}{G_{\mathrm{PCP}}} \frac{\exp \left(-\pi f_{0} t^{*}{ }_{\mathrm{PKiKP}}\right)}{\exp \left(-\pi f_{0} t_{\mathrm{PCP}}\right)}\right]$.

We converted the spectral ratios to reflection coefficients as a function of frequency. To the first order, geometric spreading is constant with respect to frequency under a ray-theoretical assumption. The reflection and transmission coefficients were then calculated for planar boundaries. The correction of the attenuation factor using $t^{*}$ gives a smooth exponential variation with frequency. 


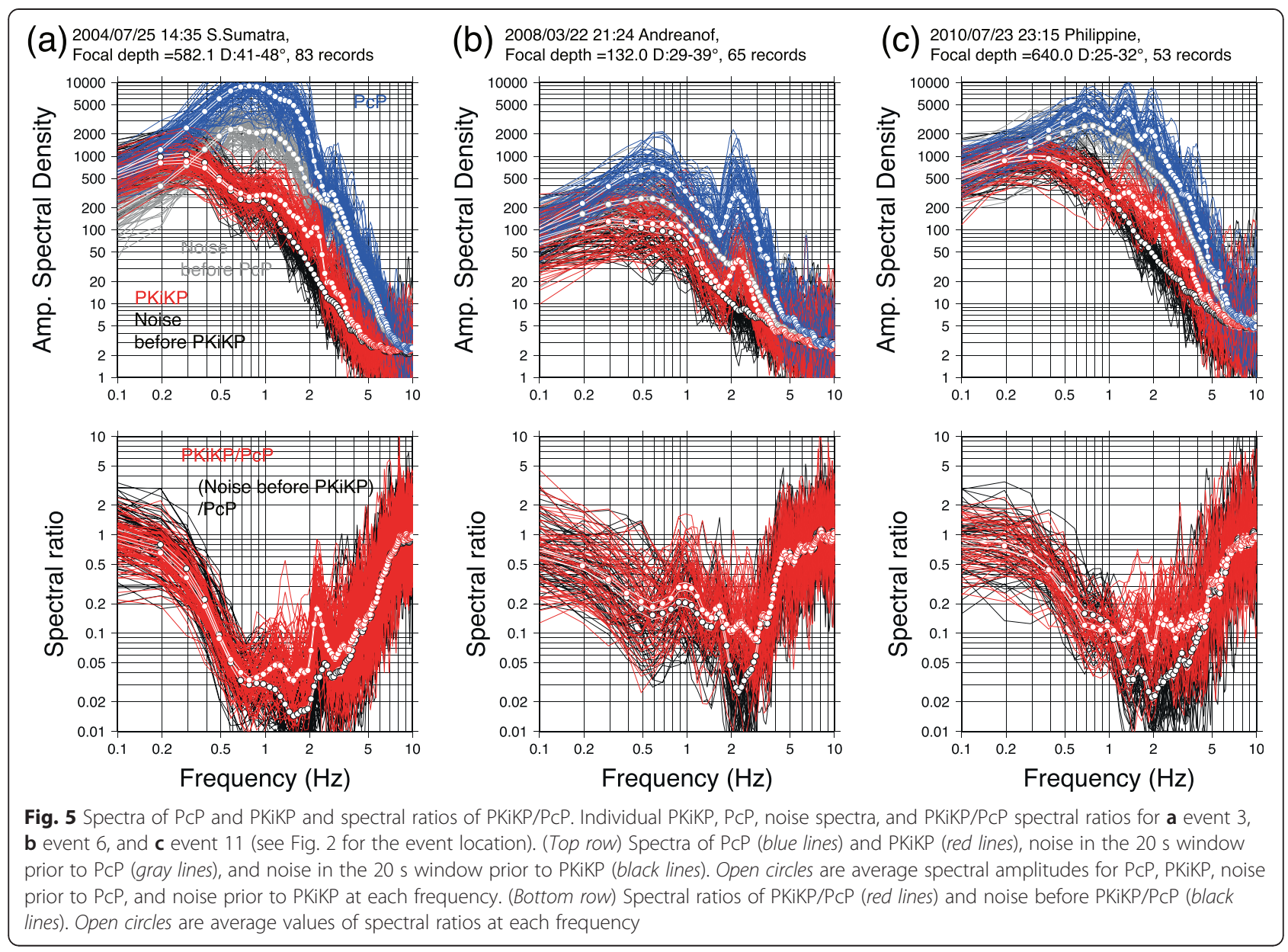

Thus, these corrections will not result in any spectral holes or peaks.

To reduce the unwanted effect of the $\mathrm{CMB}$ on the $\mathrm{PcP}$ spectra, the spectral ratios of $\mathrm{PcP} / \mathrm{P}$ were examined. Similar to the above procedure, we estimated the $\mathrm{P}$ wave reflection coefficients at the $\mathrm{CMB}$ as a function of frequency. The obtained reflection coefficients did not always coincide with theoretical values due to the uncertainty in focal mechanisms, large differences in P and PcP take-off angles, and other unknown causes. Thus, we corrected only the fluctuations in the reflection coefficients around the average values in the frequency range $1-3 \mathrm{~Hz}$. The reflection coefficients at the ICB are multiplied by the fluctuations in reflection coefficients at the CMB, which can result in either amplifying an apparently small $\mathrm{PKiKP} / \mathrm{PcP}$ due to a large peak in the PcP spectrum or reducing a large $\mathrm{PKiKP} / \mathrm{PcP}$ due to a small peak in the PcP.

\section{Results}

A summary of frequency-dependent ICB P wave reflection coefficients, as derived from Eqs. (1) and (2), is shown in Fig. 6. Panels are sorted by increasing incidence angle at the ICB; we refer to each panel hereafter as result $n$, where $n$ stands exclusively for panel number. Figure 7 summarizes frequency-dependent $\mathrm{P}$ wave reflection coefficients at the $\mathrm{CMB}$ derived from $\mathrm{PcP} / \mathrm{P}$ spectral ratios. In Fig. 8, these are used to correct the reflection coefficients at the ICB (Fig. 6). These corrected values are used in finite difference modeling and subsequent interpretation.

The frequency characteristics of the ICB reflection coefficients are quite complex, even in a narrow effective signal band (Fig. 8). Roughly speaking, peaks in reflection coefficients appear around $2 \mathrm{~Hz}$ (results 8, 9, and 10) and $3 \mathrm{~Hz}$ (result 3), and holes are observed around $1 \mathrm{~Hz}$ (results 2, 3, and 4) and $3 \mathrm{~Hz}$ (result 10). Although the discrimination is still qualitative, we recognize four general categories of frequency-dependent characteristics: (i) a flat variation, where fluctuations in the relative strengths of peaks and holes are between half and double those of the theoretical reflection coefficients (results $1,5,6,7$, and 11); (ii) a distinct single hole in each reflection coefficient spectrum (results 2 and 4); (iii) a strong single peak in each spectrum (results 8 and 9); 


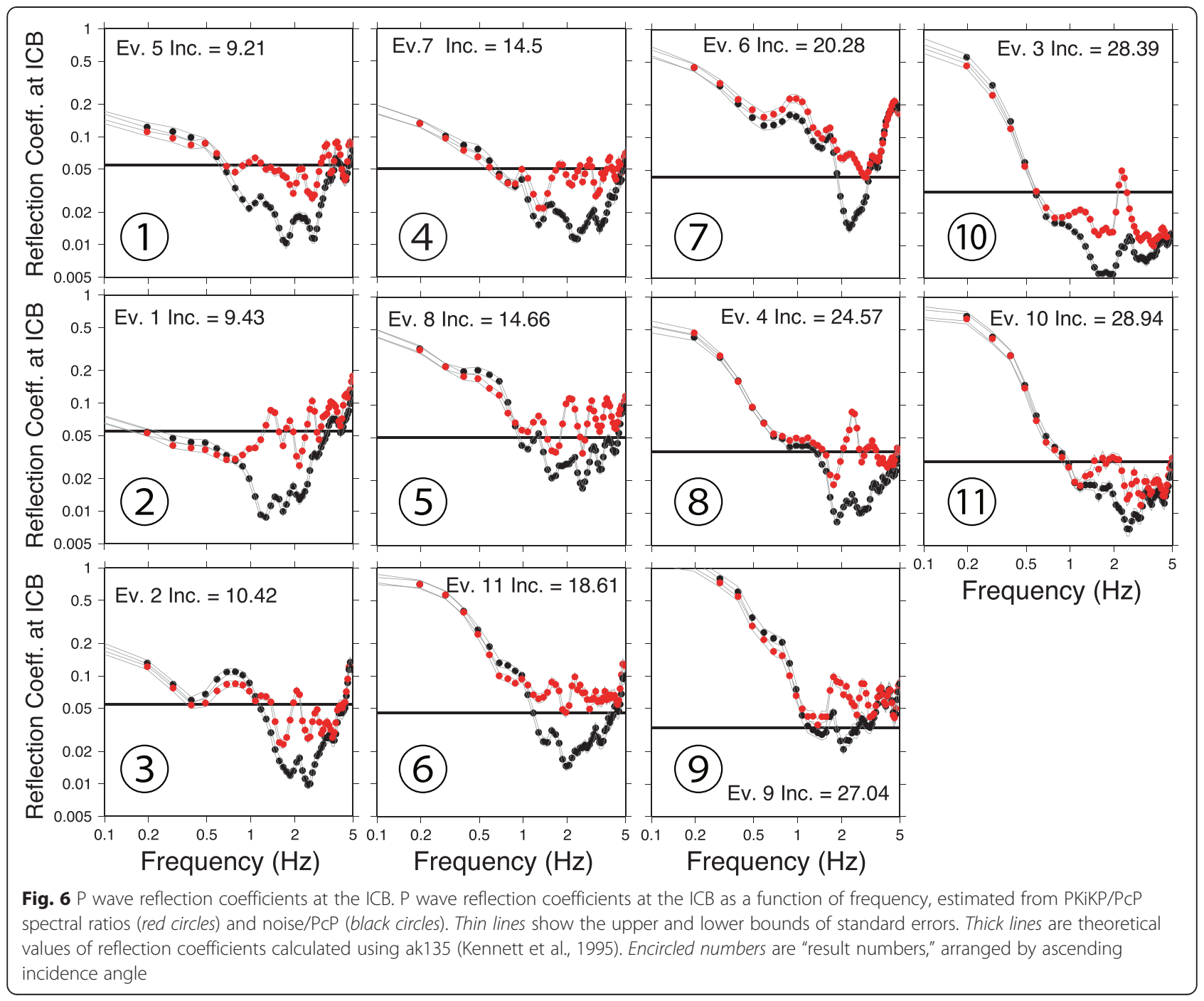

and (iv) a strong peak and hole in the same spectrum (results 3 and 10).

The geographical distribution of these categories is plotted in Fig. 9. The diameters of the 1 and $2 \mathrm{~Hz}$ Fresnel zones for PKiKP are approximately 80 and $60 \mathrm{~km}$ at the ICB, respectively. This is equivalent to the area of PKiKP reflection points at the ICB that is covered by a single event and all stations that detect PKiKP. This result suggests that our averaging within each region number is meaningful, but it is not appropriate in discussing lateral variations in individual measurements within each region. Although the sampling areas are sparse, we note a tendency of frequency peaks in reflection coefficient spectra to be most observable at low latitudes (results 8 , 9, and 10). Frequency holes show no such latitudinal trend (results 2, 3, 4, and 10).

\section{Discussion}

\section{Effects of the CMB}

Regarding the effects of the CMB on PKiKP spectra during transmission through the $\mathrm{CMB}$, we address this issue in the context of the results of previous studies. Using amplitude of precursors to PKIKP, Dai et al. (2012) and Yao and Wen (2014) showed that several regions exhibit weak scattering in the lowermost mantle beneath the southwestern Pacific. PKiKP phases from events that occurred in the Banda Sea (events 4 and 9), Sumatra (events 3 and 10), and the Philippines (event 11) enter a "normal" CMB. According to Hedlin and Shearer (2000), there is a relatively weak scattering region in the lowermost mantle beneath the Philippine Sea, which corresponds to the CMB entry points of PKiKP for events 1 and 5 and the $\mathrm{CMB}$ exit points for events 1, 3, 4, 5, 9, 


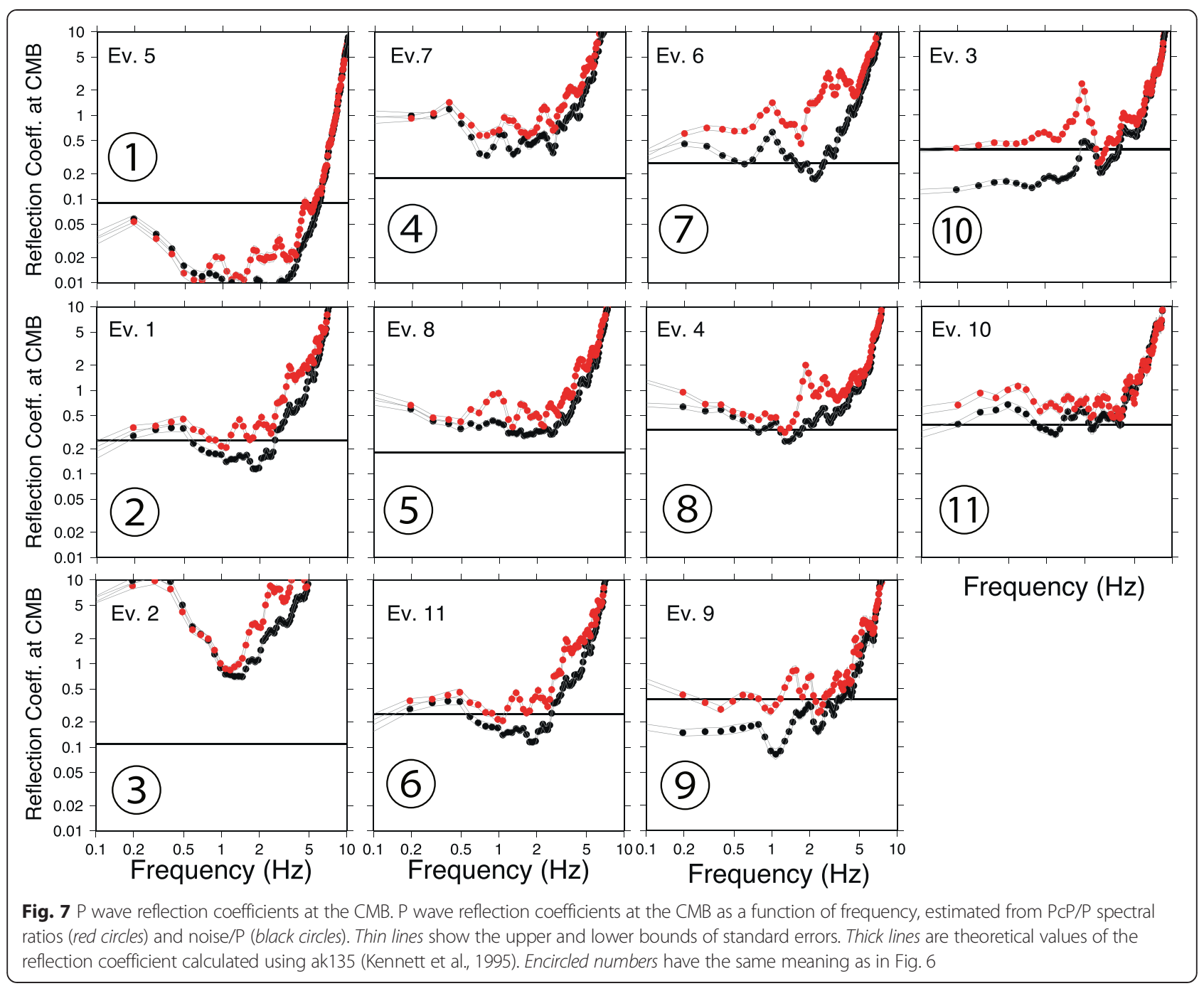

10, and 11. Thus, we have reason to believe that the CMB effects on the estimated reflection coefficients for results $1,2,6,8,9,10$, and 11 will be negligible. However, a strong scattering area near the CMB exists beneath north Japan and the northwestern Pacific, which includes the PKiKP CMB entry and exit points for events $2,6,7$, and 8 . Thus, we cannot rule out the possibility that the frequency characteristics of calculated reflection coefficients for results $3,4,5$, and 7 are $\mathrm{CMB}$ effects, e.g., the high-frequency components of PKiKP may be lost by scattering at the CMB.

\section{Numerical simulations}

\section{Problem setup}

To explain our observations, we use the $2 \mathrm{D}$ finite difference program e3d (Larsen and Shultz 1995; Rodgers et al. 2006) to simulate wave propagation by solving the full wave equation on a staggered grid. The solutions are fourth order accurate in space and second order accurate in time. Figure 10a shows the configuration of the simulation, for which the grid spacing is $70 \mathrm{~m}$. P- and Swave velocities and densities above and below the ICB are taken from ak135 (Kennett et al. 1995). We place a sequence of point sources with $1 \mathrm{~km}$ spacing on a straight line $100 \mathrm{~km}$ long and generate a plane wave. The incidence angle $\theta$ is a control parameter. As the input, the representative $\mathrm{P}$ wave waveform is taken from the Mariana event (event 1). The calculation is valid for frequencies up to $5 \mathrm{~Hz}$. We examine the observed spectral ratios between incident and reflected waves at three points (triangles in Fig. 10a). To verify the configuration and boundary conditions, and to ensure that there are no unwanted numerical effects from the edges of the box, we conducted a test run of a simple, flat ICB, with elastic parameters on both sides of the ICB taken from the ak135 model (Fig. 10b). The spectral ratios between upgoing and downgoing waves for incidence angles of $10^{\circ}, 20^{\circ}$, and $30^{\circ}$ correspond to epicentral distances of approximately $16^{\circ}, 32^{\circ}$, and $48^{\circ}$, respectively. Although there are small frequency-dependent fluctuations (within 


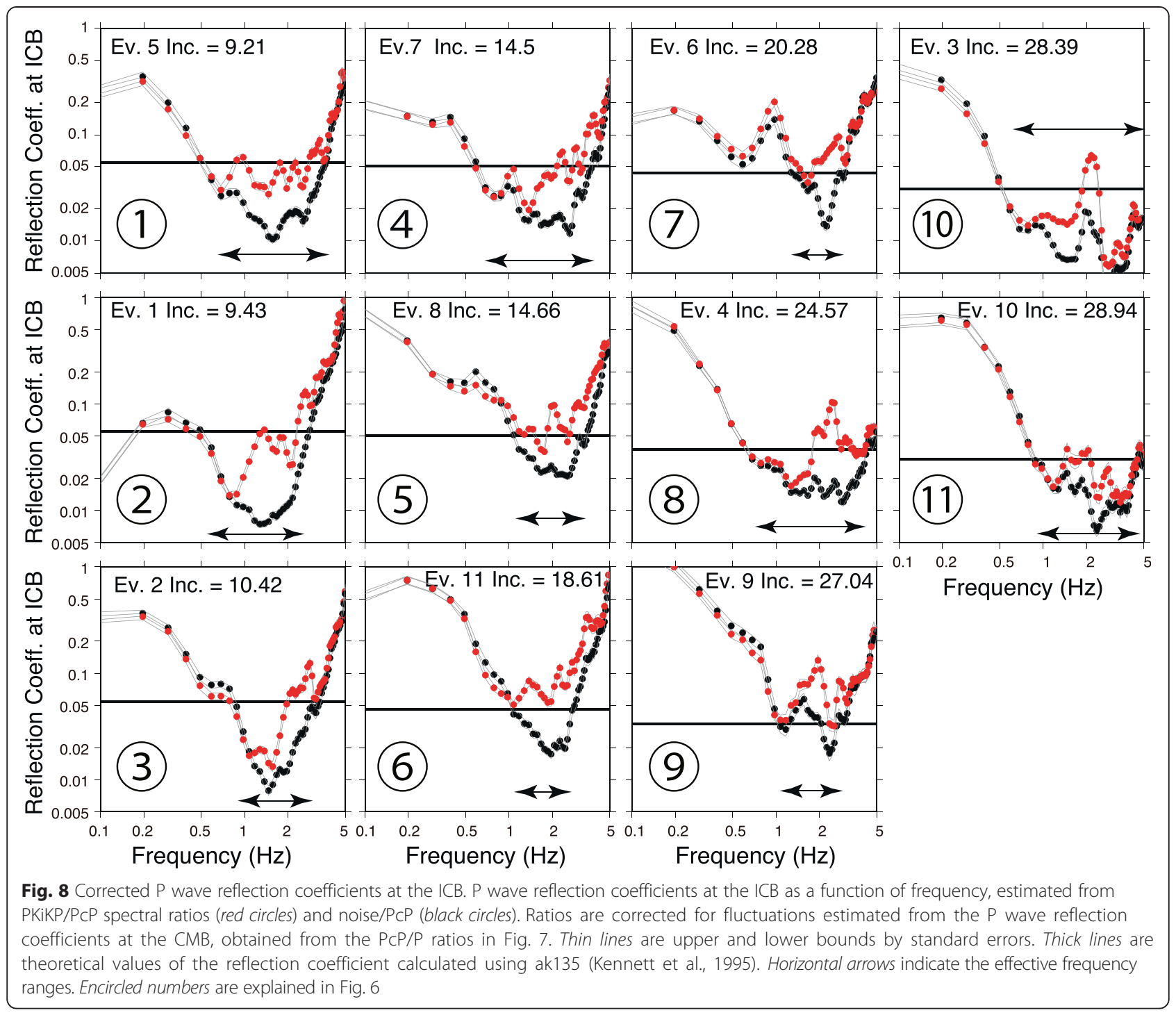

a factor of 2, at most), their average values coincide well with the theoretical reflection coefficients at the ICB.

\section{Simulations for a thin layer above the ICB}

As discussed in the "Background" section, there are multiple conceptual models that can explain freezing and melting of the quasi-eastern hemisphere. To investigate the possibility of a thin layer above the ICB, we run a series of simulations with layer thicknesses of $0.1,0.25,0.5,0.75,1.0,3.0$, and $5.0 \mathrm{~km}$. For a solid layer above the ICB with finite shearwave velocity and slight variations in compressional wave velocity and density $(\mathrm{Vp}=10.6-10.9 \mathrm{~km} / \mathrm{s}, \mathrm{Vs}=$ $\left.1-3 \mathrm{~km} / \mathrm{s}, \quad \rho=12.4-12.5 \mathrm{~g} / \mathrm{cm}^{3}\right)$, all the resultant reflected wave amplitudes are small. However, a light or heavy liquid layer $(\mathrm{Vp}=9.6-10.3 \mathrm{~km} / \mathrm{s}, \rho=12.5 \mathrm{~g} /$ $\mathrm{cm}^{3}$ for heavy liquid, $\rho=11.5 \mathrm{~g} / \mathrm{cm}^{3}$ for light liquid) results in large amplitudes. In particular, we find that the amplitudes of seismograms filtered with central frequencies of 1 and $2 \mathrm{~Hz}$ become large when we insert a heavy liquid layer above the ICB with $\mathrm{Vp}=9.6 \mathrm{~km} / \mathrm{s}, \rho=12.5 \mathrm{~g} / \mathrm{cm}^{3}$, and a thicknesses of 0.75 or $1 \mathrm{~km}$ (Fig. 11a, b). This finding is qualitatively consistent with the results of Krasnoshchekov et al. (2005). However, we also find that spectral ratios continuously increase as a function of frequency for any incidence angle (Fig. 11c), without distinct peaks or holes in the frequency range 0.5 to $3 \mathrm{~Hz}$. Since this does not explain our observations, we reject the model of a thin layer above the ICB as a possible explanation for the observed frequencydomain characteristics of PKiKP. 


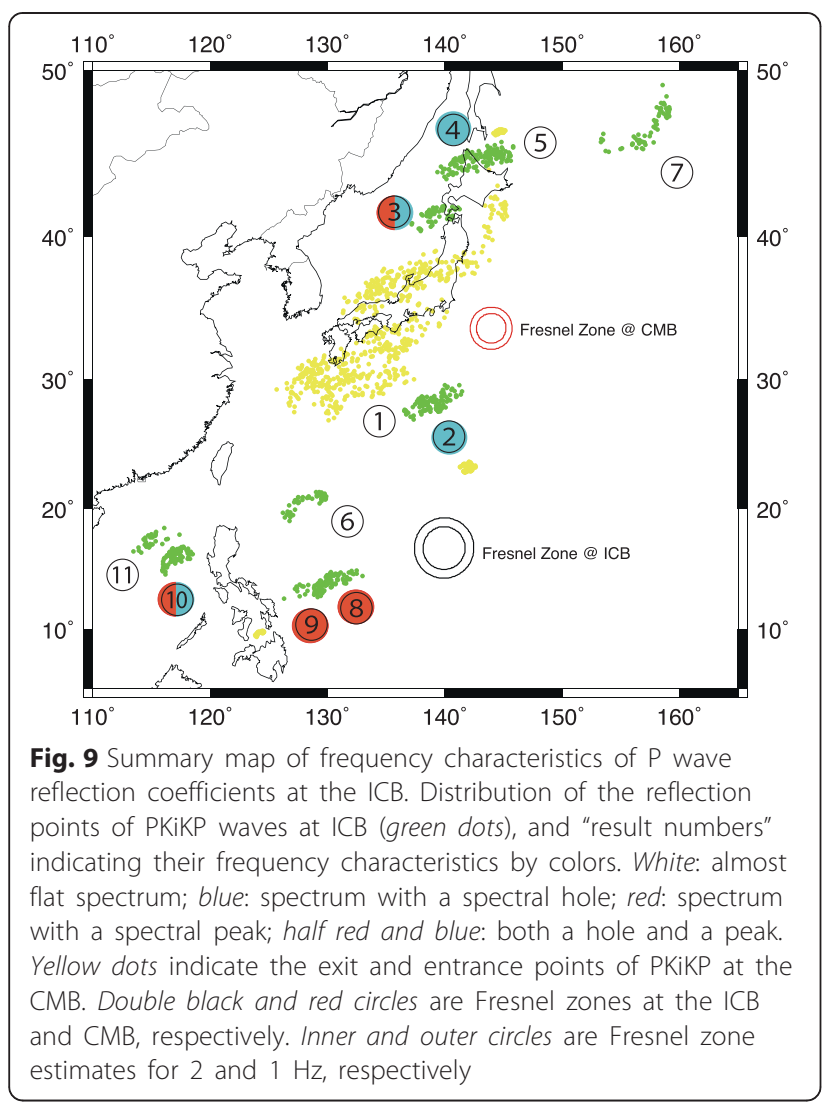

\section{Simulations for topography at the ICB}

Earlier PKiKP and PcP amplitude ratio analyses made sporadic seismological observations of a lower density contrast at the ICB than predicted values for spherically symmetric Earth models, as low as 200-300 $\mathrm{g} / \mathrm{m}^{3}$ (Koper and Pyle 2004; Tkalčić et al. 2009). In addition, Gubbins et al. (2008) inferred a low density contrast from geodynamical considerations. These results indirectly support the existence of a dense layer at the top of the inner core (F-layer) to explain the smaller density differences between the outer and inner cores. However, there is no proof from seismology that such a layer is a global feature. Masters and Gubbins (2003) noted that even the relatively large density jump inferred from free oscillation analyses is consistent with an F-layer having a strong density gradient: the free oscillation data would average over thick layers below and above the ICB, while the body wave would be sensitive to variations across the ICB. A lower density contrast across the ICB would permit larger topography (Buffett 1997). Of particular interest is the possibility of sharp edges between the solidification and melting areas. Recent waveform modeling suggests significant topography
(Dai et al. 2012); however, determination of the amplitude is a more difficult problem than determination of the wavelength.

One possibility of most simplified geometry is that a sinusoidal topographic structure might develop at the largest scales $(\lambda=10-100 \mathrm{~km})$ (Buffett 1997). At these length scales, there is an inverse relationship between relaxation time scale and wavelength (Turcotte and Schubert 2002). In addition, the time scale required for topography to relax varies inversely with density contrast and linearly with viscosity. As the viscosity of the outer core is effectively zero (de Wijs et al. 1998), the rate of relaxation is thus entirely controlled by deformation in the inner core.

On the other hand, spike-shaped topographic structures might develop as a result of dendritic growth, likely at smaller scales $(\lambda=10-$ several $100 \mathrm{~m})$ (Bruce Buffett, pers. comm.). Such topography could be relaxed through melting and freezing (thermal relaxation). As the temperature gradients are steeper at short wavelengths, this can drive the heat flow needed to melt or freeze.

Given the above, we test two different classes of topography at the ICB. In the first scenario, we test a regular sinusoidal topography with wavelength $\lambda$ and height $H$ (Fig. 12a). The values of $(\lambda, H)$ tested, in kilometers, are (0.1, 0.1), (0.2, 0.2), (0.5, 0.5), (1.0, 1.0), (1.5, 1.5), (2.5, $2.5),(2.5,2.6),(2.5,2.7),(5.0,2.5)$, and $(10.0,5.0)$. Figure 13 shows the results of selected combinations of $\lambda$ and $H$. The topographies with $\lambda=0.1$ and $0.2 \mathrm{~km}$ and $H=0.1$ and $0.2 \mathrm{~km}$ did not reproduce any spectral peaks and holes and yielded spectra similar to those obtained from a simple discontinuity model (Fig. 10b). However, for a slightly more prominent topography of $\lambda=0.5 \mathrm{~km}$ and $H=0.5 \mathrm{~km}$, we find a clear peak in spectral ratios around $1.2 \mathrm{~Hz}$ (Fig. 13a). The ICB topography with $\lambda=1.0$ and $H=1.0 \mathrm{~km}$ results in an increased number of spectral peaks (Fig. 13b). The topographic model with $\lambda=1.5$ and $H=1.5 \mathrm{~km}$ produces distinct peaks around $2 \mathrm{~Hz}$ for incidence angles of $20^{\circ}$ and $30^{\circ}$ and remarkable holes around $1.2 \mathrm{~Hz}$ for incidence angles of $10^{\circ}$ and $30^{\circ}$ (Fig. 13c). The best results are obtained for the topography characterized by $\lambda=1.5$ and $H=1.5 \mathrm{~km}$. The simulated spectra contain similar characteristics to those observed in the reflection coefficient profiles of results 8,9 , and 10 . However, when the longer $\lambda$ and larger $H$ are used in simulations (e.g., $\lambda=$ 5 and $H=2.5 \mathrm{~km} ; \lambda=10$ and $H=5 \mathrm{~km}$ ), the reflection coefficients overall become smaller than those for the flat ICB. The spectra are devoid of peaks at higher frequencies (Fig. 13d, e).

Finally, we introduce "spiky" topography to address the possibility of dendritic growth of the inner core (Sumita and Bergman 2009), which is mathematically expressed in our simulations by a reversed cycloid with 
(a)

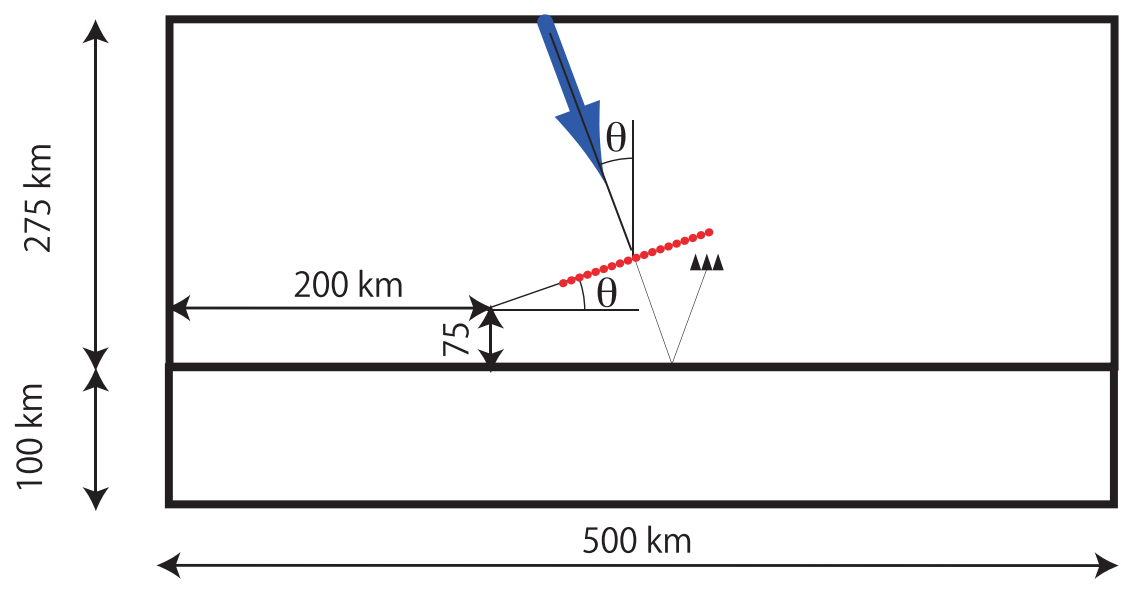

(b)

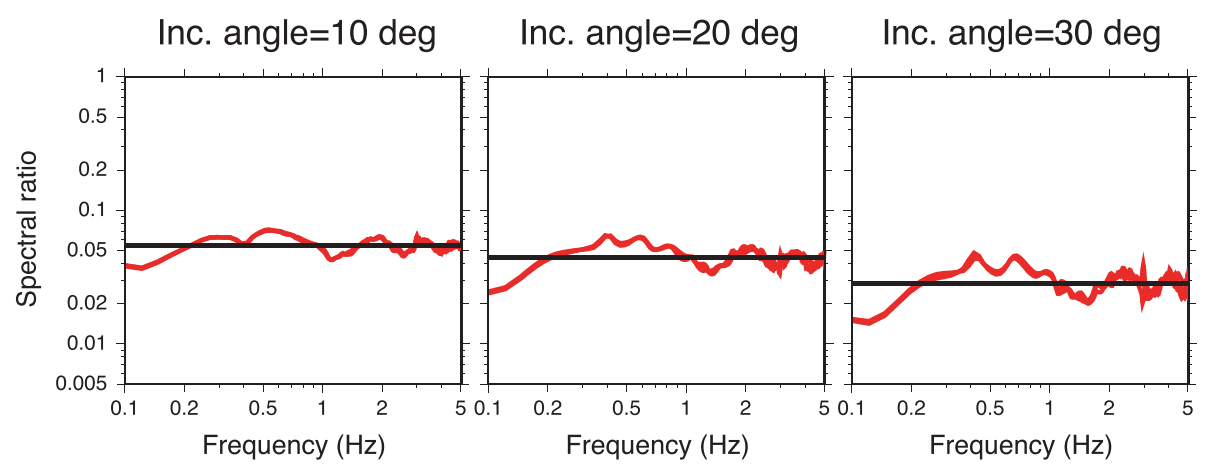

Fig. 10 Configuration of 2D finite difference simulations and a test run. a Configuration of 2D finite difference simulations using e3d (Larsen and Shultz 1995). Small red circles represent multiple sources used to simulate a plane wave. Triangles mark the locations of virtual receivers used to record the reflected waves. Incidence angles $\theta$ are a control parameter. b Spectral ratios between upward and downward waves using a flat discontinuity in simulations for incidence angles of $10^{\circ}, 20^{\circ}$, and $30^{\circ}$. Thick lines are theoretical values of the reflection coefficient calculated using ak135 (Kennett et al. 1995)

upward sharp tips (Fig. 12b). We tested several $(\lambda, H)$ combinations for this conceptual model: $(0.1,0.1),(0.2$, $0.2)$, (0.5, 0.2), (0.5, 0.1), (0.5, 0.2), (1.0, 0.2), (1.0, 0.5), (1.0, 1.0), (1.0, 2.0), (1.2, 1.7), (1.5, 1.5), (1.5, 2.0), (2.0, $1.0),(2.0,1.5),(2.0,2.0)$, and $(5.0,2.0)$. The overall characteristics of the spectral ratios for spiky topography, with $\lambda \leq 2 \mathrm{~km}$ and $H \leq 2 \mathrm{~km}$ (Fig. 14a-d), can be summarized as a gradual decrease in spectral ratio as frequency increases from 0.5 to $5 \mathrm{~Hz}$. This topographic model can explain several peaks or holes for incidence angles of $\theta=10^{\circ}$ and $20^{\circ}$ and many peaks at frequencies around 2,3 , and $4 \mathrm{~Hz}$ for $\theta=30^{\circ}$. The topography with $\lambda=5 \mathrm{~km}$ and $H=2 \mathrm{~km}$ results in relatively flat and small spectral ratios in the frequency range $0.7-5 \mathrm{~Hz}$. Such a model can explain many holes at frequencies larger than $2.5 \mathrm{~Hz}$ for $\theta=10^{\circ}-20^{\circ}$ and peaks for $\theta=30^{\circ}$. However, these results cannot adequately explain our observations because the overall reflection coefficients are too small.
The resultant characteristics are slightly different from those produced by the sinusoidal topographies, even though their structural dimensions are the same. The frequencies of the distinct spectral peaks decrease with increasing wavelength and height (Fig. 14b-d), whereas no distinct peaks are observed for the topography with $\lambda=H=0.5 \mathrm{~km}$. The spiky topography with $\lambda=H=$ $1.0 \mathrm{~km}$ results in a distinct peak around $1.7 \mathrm{~Hz}$ for $\theta=$ $10^{\circ}-20^{\circ}$ and a large hole around $1.5 \mathrm{~Hz}$ for $\theta=30^{\circ}$ (Fig. 14b). The topography with $\lambda=1.5$ and $H=1.5 \mathrm{~km}$ yields a distinct single peak at roughly $f=1.2 \mathrm{~Hz}$. There are several spectral holes for $\theta=10^{\circ}-20^{\circ}$ and peaks for $\theta=30^{\circ}$ at $f>2 \mathrm{~Hz}$ (Fig. 14c). Of all the test cases with spiky topography, the case with $\lambda=H=1.0 \mathrm{~km}$ most closely matches the observed spectral ratios.

In summary, our observations and numerical simulations suggest that the inner core boundary is a sharp boundary without transitional layers. The hypothesis of 
(a)

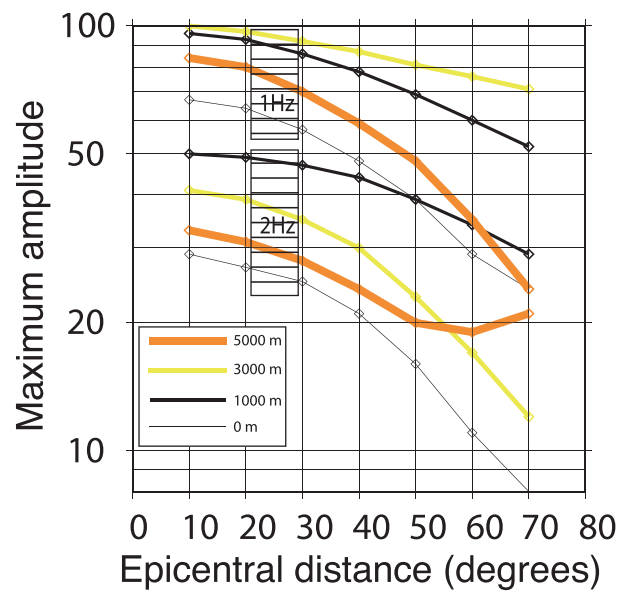

(b)

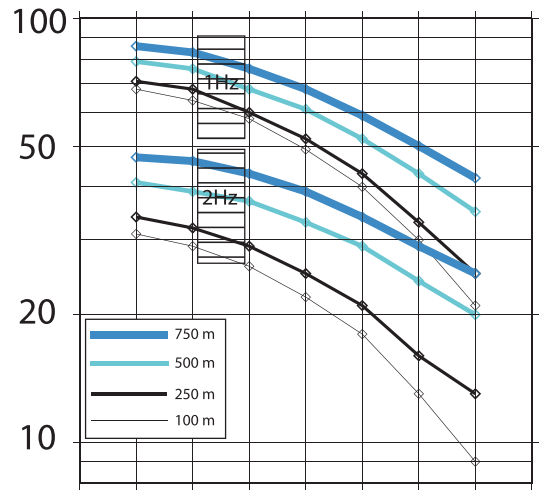

01020304050607080

Epicentral distance (degrees)

(c)

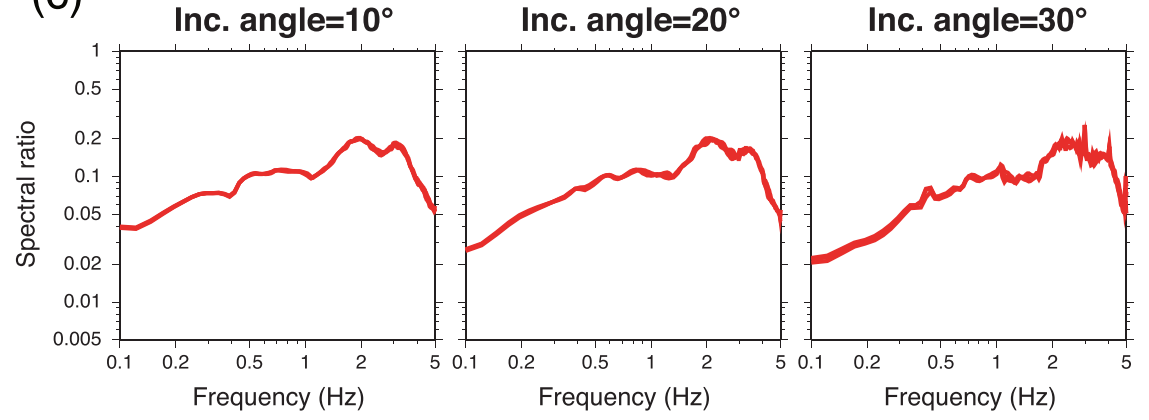

Fig. 11 Results of numerical simulations for a liquid thin layer above the ICB. Maximum amplitudes of the reflected waves measured on seismograms filtered around 1 and $2 \mathrm{~Hz}$ as a function of increasing angular distance at the ICB. Synthetic seismograms are calculated by including a heavy liquid layer $\left(V p=9.6 \mathrm{~km} / \mathrm{s}, \rho=12.5 \mathrm{~g} / \mathrm{cm}^{3}\right)$ overlying the ICB with varying thicknesses: a $0,1000,3000$, and $5000 \mathrm{~m}$ and b $100,250,500$, and $750 \mathrm{~m}$. c Spectral ratios for the $1000 \mathrm{~m}$-thick heavy liquid layer for incidence angles of $10^{\circ}, 20^{\circ}$, and $30^{\circ}$

(a)

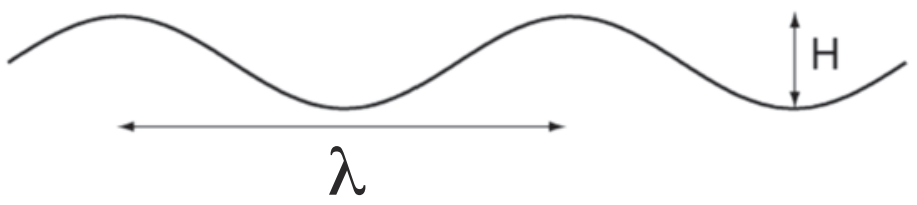

(b)

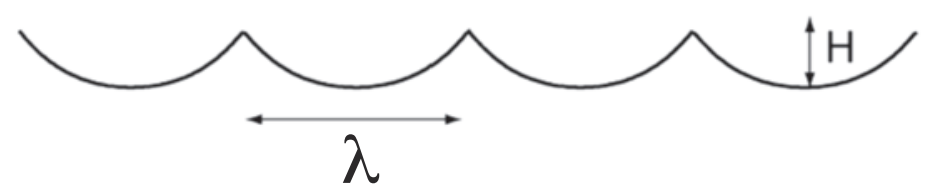

Fig. 12 Diagram of ICB topography. Diagram of ICB topography used in numerical simulations with wavelength and height defined as $\lambda$ and $H$, respectively. a Sinusoidal topography; b "spiky" topography. The meaning of each topographic model is explained in the text 


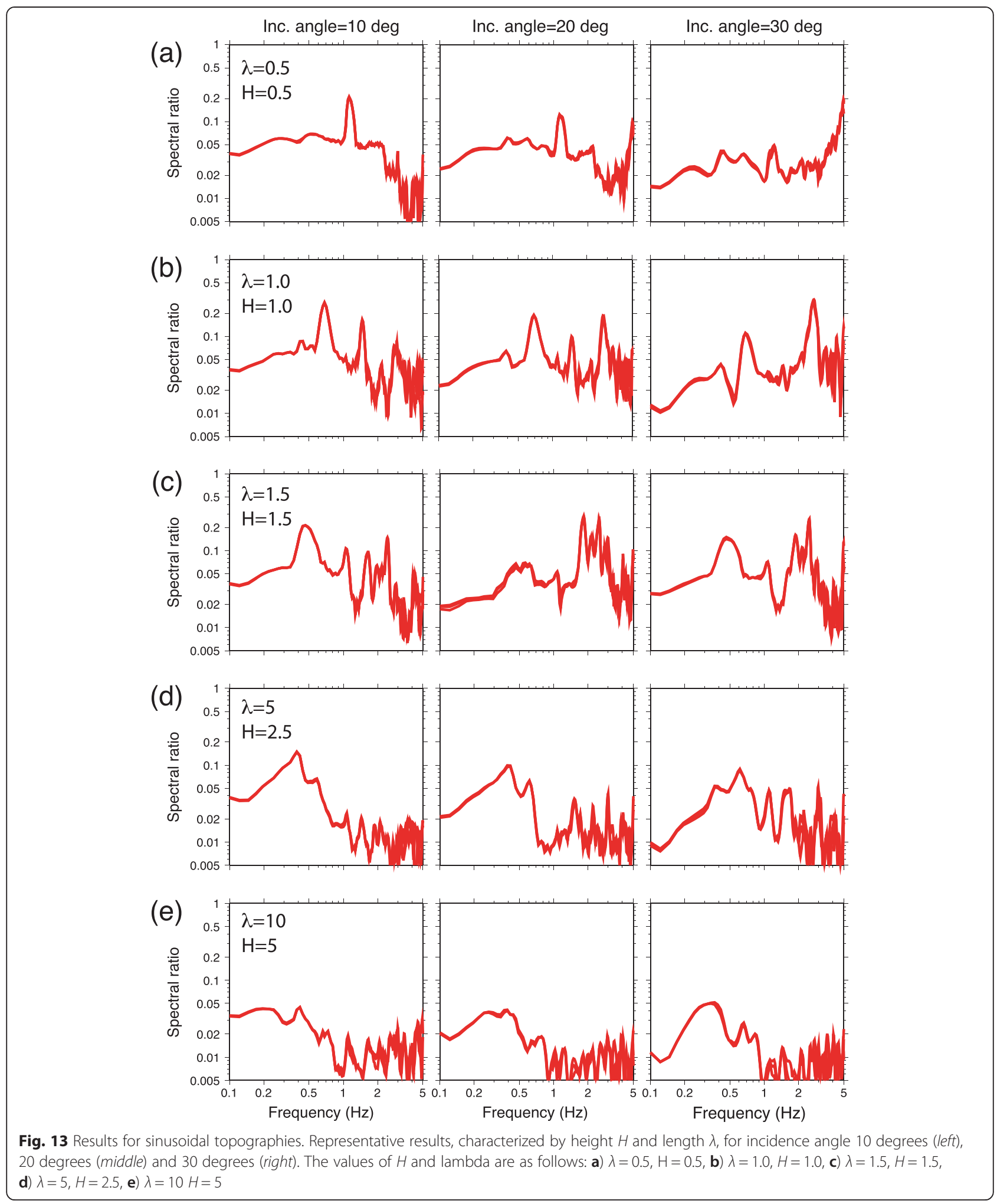

melt at the surface of the inner core in the quasi-eastern hemisphere is not supported by our simulations. The most likely scenario to explain some of the observed spectral characteristics is the existence of topography at the ICB; however, more than one class of topography must be invoked to explain all observations. We therefore conclude that the topography characteristics of the ICB vary laterally. These variations may result from 


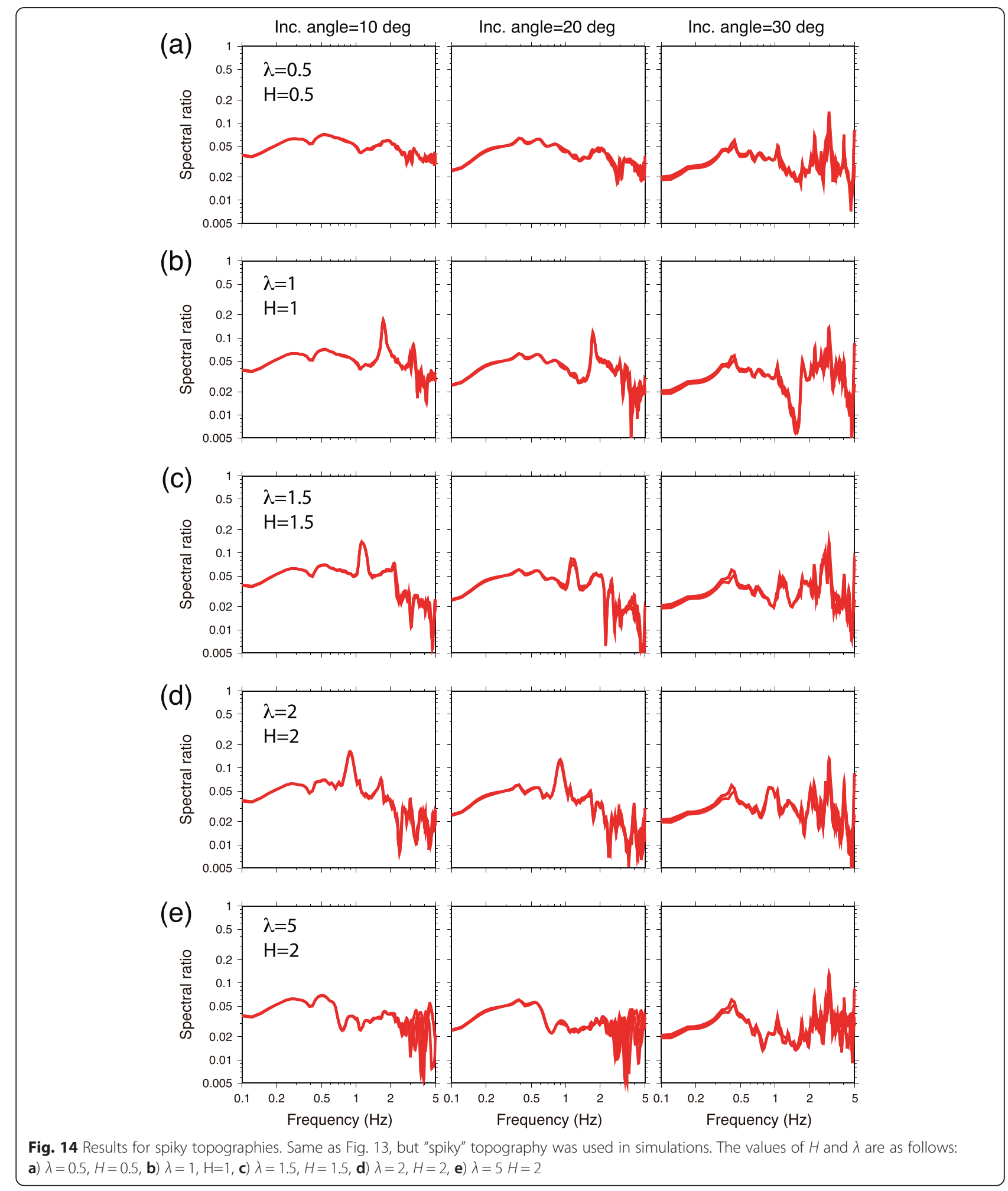

lateral variations of inner core solidification. If solidification is dynamically driven from top to bottom, its geographical pattern will be controlled by the pattern of outer core convection (Bergman et al. 2002; Aubert et al. 2008; Gubbins et al. 2011). If the solidification is instead driven from the bottom up, the pattern will be affected by variations in inner core convection (Deguen and Cardin 2011). Furthermore, small-scale variations in topographic characteristics suggest small-scale convection in a mushy zone at the ICB (Bergman and Fearn 
1994; Deguen et al. 2007). While we cannot distinguish between these hypotheses in the present study, largely due to the fact that we sample only sparse and limited areas of the ICB, further observations of PKiKP and PcP will improve our understanding of large-scale ICB structure and dynamics.

\section{Conclusions}

Frequency characteristics of ICB reflection coefficients were investigated for the area around Japan using Hi-net vertical component seismograms. We found four patterns in the frequency-dependent behavior of reflection coefficients: (a) a nearly flat spectrum (little variation), (b) a significant hole at a frequency of approximately 1 or $3 \mathrm{~Hz}$, (c) a peak at a frequency of approximately 2 or $3 \mathrm{~Hz}$, and (d) the existence of a hole and a peak. The variety in observed spectra reflects the complex nature of the ICB. To interpret these observations, we conducted 2D finite difference simulations. Since we tested only limited cases with planar geometry, further simulations are required. Our modeling results suggest that holes and peaks in the spectra of reflection coefficients can be qualitatively explained by a sinusoidal or spikelike topography at the ICB, with wavelengths and heights $\sim 1-1.5 \mathrm{~km}$, whereas a liquid or solid layer overlying the ICB does not reproduce any of the observed spectral features.

\section{Abbreviations \\ CMB: core-mantle boundary; ICB: inner core boundary.}

\section{Competing interests}

The authors declare that they have no competing interests.

\section{Authors' contributions}

ST analyzed waveform data. HT performed finite difference simulations. The manuscript was written by ST and HT. Both authors read and approved the final manuscript.

\section{Authors' information}

ST is Deputy Director and Senior Scientist at the Department of Deep Earth Structure and Dynamics Research, Japan Agency for Marine-Earth Science and Technology.

HT is Associate Professor and Senior Fellow at Seismology and Mathematical Geophysics, Research School of Earth Sciences, The Australian National University.

\section{Acknowledgements}

The authors are grateful to the National Research Institute for Earth Science and Disaster Prevention (NIED), Japan, for providing high-quality seismograms recorded by the high-sensitivity seismograph network (Hi-net). The authors thank two anonymous reviewers who give valuable comments. S.T. was supported in part by MEXT KAKENHI grant number 15H05832. H.T. was supported by the Japan Society for the Promotion of Science and the Research School of Earth Science, The Australian National University during his stay in Japan. Most of the figures were drawn using GMT (Wessel and Smith 1998).

\section{Author details}

'Department of Deep Earth Structure and Dynamics Research, Japan Agency for Marine-Earth Science and Technology, Yokosuka 237-0061, Japan. ${ }^{2}$ Research School of Earth Sciences, The Australian National University, Canberra ACT 2601, Australia.
Received: 21 April 2015 Accepted: 8 October 2015

Published online: 04 November 2015

\section{References}

Alboussiere T, Deguen R, Melzani M (2010) Melting-induced stratification above the Earth's inner core due to convective translation. Nature 466:744-9

Aubert J, Amit H, Hulot G, Olson P (2008) Thermochemical flows couple the Earth's inner core growth to mantle heterogeneity. Nature 454:758-62

Bergman MI, Fearn DR (1994) Chimneys on the Earth's inner-outer core boundary. Geophys Res Lett 21:477-80

Bergman MI, Cole DM, Jones JR (2002) Preferred crystal orientations due to melt convection during directional solidification. J. Geophys. Res., 107: doi:10.1029/ 2001JB000601

Buchbinder GGR (1972) Travel times and velocities in the outer core from PmKP. Earth Planet Sci Lett 14:161-8

Buffett BA (1997) Geodynamic estimates of the viscosity of the Earth's inner core. Nature 388:571-3

Cao A, Romanowicz B (2004) Constraints on density and shear velocity contrast at the inner core boundary. Geophys J Int 157:1146-51

Cormier VF (2007) Texture of the uppermost inner core from forward- and backscattered seismic waves. Earth Planet Sci Lett 258:442-53

Creager KC (1999) Large-scale variations in inner core anisotropy. J Geophys Res 104:23127-39

Cummins P, Johnson L (1988) Synthetic seismograms for an inner core transition of finite thickness. Geophys J 94:21-34

Dai Z, Wang W, Wen L (2012) Irregular topography at the Earth's inner core boundary. Proc Natl Acad Sci 109:7654-8

de Wijs GA, Kresse G, Vocadlo L, Dobson DP, Alfè D, Gillan M, Price GD (1998) The viscosity of liquid iron at the physical conditions of the Earth's core. Nature 392:805-7

Deguen R (2012) Structure and dynamics of Earth's inner core. Earth Planet Sci Lett 333-334:211-25

Deguen R, Cardin P (2011) Thermochemical convection in Earth's inner core. Geophys J Int 187:1101-18

Deguen R, Alboussiere T, Brito D (2007) On the existence and structure of a mush at the inner core boundary of the Earth. Phys Earth Planet Inter 164:36-49

Deuss A (2014) Heterogeneity and anisotropy of Earth's inner core. Ann Rev Earth Planet Sci 42:103-26

Deuss A, Irving JCE, Woodhouse JH (2010) Regional variation of inner core anisotropy from seismic normal mode observations. Science 328:1018-20

Engdahl ER, Flinn EA, Romney CF (1970) Seiemic waves reflected from the Earth's inner core. Nature 228:852-3

Engdahl ER, Flinn EA, Massé RP (1974) Differential PKiKP travel times and the radius of the inner core. Geophys J R Astron Soc 39:457-63

Gubbins D, Masters G, Nimmo F (2008) A thermochemical boundary layer at the base of Earth's outer core and independent estimate of core heat flux. Geophys J Int 174:1007-18

Gubbins D, Sreenivasan B, Mound J, Rost S (2011) Melting of the Earth's inner core. Nature 473:361-3

Hedlin MAH, Shearer PM (2000) An analysis of large-scale variations in small-scale mantle heterogeneity using global seismographic network recordings of precursors to PKP. J Geophys Res 105:13655-73

Jiang G, Zhao D (2012) Observation of high-frequency PKiKP in Japan: insight into fine structure of inner core boundary. J Asian Earth Sci 59:167-84

Kawakatsu H (2006) Sharp and seismically transparent inner core boundary region revealed by an entire network observation of near-vertical PKiKP. Earth Planets Space 58:855-63

Kennett BLN, Engdahl ER, Buland R (1995) Constraints on seismic velocities in the Earth from travel-times. Geophys I Int 122:108-24

Kikuchi M, Kanamori H (2003), Note on teleseismic body-wave inversion program, http://www.eri.u-tokyo.ac.jp/ETAL/KIKUCHI/

Koper KD, Dombrovskaya M (2005) Seismic properties of the inner core boundary from PKiKP/P amplitude ratios. Earth Planet Sci Lett 237:680-94

Koper KD, Pyle ML (2004) Observations of PKiKP/PcP amplitude ratios and implications for Earth structure at the boundaries of the liquid core. J. Geophys. Res., 109: doi:10.1029/2003JB002750

Koper KD, Pyle ML, Franks JM (2003) Constraints on aspherical core structure from PKiKP-PcP differential travel times. J Geophys Res 108:2168, doi:2110.1029/2002JB001995 
Koper KD, Franks JM, Dombrovskaya M (2004) Evidence for small-scale heterogeneity in Earth's inner core from a global study of PKiKP coda waves. Earth Planet Sci Lett 228:227-41

Krasnoshchekov DN, Kaazik PB, Ovtchinnikov VM (2005) Seismological evidence for mosaic structure of the surface of the Earth's inner core. Nature 435 $483-7$

Larsen SC, Shultz CA (1995), E3D:2D/3D Elastic finite-difference wave propagation code, Lawrence Livermore National Laboratory,Livermore, CA, USA. 1-18.

Lay T, Wallace T (1995) Modern global seismology. Academic, San Diego

Leyton F, Koper KD (2007a) Using PKiKP coda to determine inner core structure: 1. Synthesis of coda envelopes using single-scattering theories. J. Geophys. Res., 112: doi:10.1029/2006JB004369.

Leyton F, Koper KD (2007b) Using PKiKP coda to determine inner core structure:

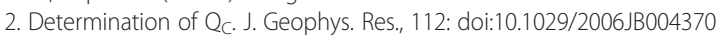

Leyton F, Koper KD, Zhu L, Dombrovskaya M (2005) On the lack of seismic discontinuities within the inner core. Geophys J Int 162:779-86

Loper DE (1983) Structure of the inner core boundary. Geophys Astrophys Fluid Dyn 25:139-55

Loper DE, Roberts PH (1981) A study of conditions at the inner core boundary of the Earth. Phys Earth Planet Inter 24:302-7

Lythgoe KH, Deuss A, Rudge JF, Neufeld JA (2014) Earth's inner core: Innermost inner core or hemispherical variations? Earth Planet. Sci Lett 385:181-9

Masters G, Gubbins D (2003) On the resolution of density within the Earth. Phys Earth Planet Inter 140:159-67

Monnereau M, Calvet M, Margerin L, Souriau A (2010) Lopsided growth of Earth's inner core. Science 328:1014-7

Niu FL, Wen LX (2001) Hemispherical variations in seismic velocity at the top of the Earth's inner core. Nature 410:1081-4

Okada Y, Kasahara K, Hori S, Obara K, Sekiguchi S, Fujiwara H, Yamamoto A (2004) Recent progress of seismic observation networks in Japan -Hi-net, F-net, KNET and KiK-net-. Earth Planets Space, 56: XV-Xxviii

Peng ZG, Koper KD, Vidale JE, Leyton F, Shearer P (2008) Inner-core fine-scale structure from scattered waves recorded by LASA. J. Geophys. Res., 113: doi:10.1029/2007jb005412

Poupinet G, Kennett BLN (2004) On the observation of high frequency PKiKP and its coda in Australia. Phys Earth Planet Inter 146:497-511

Press WH, Teukolsky SA, Vetterling WT, Flannery BP (1988) Numerical recipes in C: the art of scientific computing. Cambridge University Press, Cambridge

Rodgers A, Tkalčić H, McAllen D (2006) Seismic ground motion and site response in Las Vegas Valley, Nevada from NTS explosions and earthquake data. Pure Appl Geophys 163:55-80

Schweitzer J, Kværna T (1999) Influence of source radiation patterns on globally observed short-period magnitude estimates $\left(m_{b}\right)$. Bull Seism Soc Am 89: $342-7$

Shearer P, Masters G (1990) The density and shear velocity contrast at the inner core boundary. Geophys J Int 102:491-8

Shimizu H, Poirier JP, Le Mouël ل」 (2005) On crystallization at the inner core boundary. Phys Earth Planet Inter 151:37-51

Souriau A (2007) Deep Earth structure — the Earth's cores. In: Romanowicz B, Dziewonski AM (eds) Treatise on geophysics, vol. 1, seismology and structure of the Earth. Elsevier, Amsterdam, pp 655-93

Souriau A, Souriau M (1989) Ellipticity and density at the inner core boundary from subcritical PKiKP and PCP data. Geophys J Int 98:39-54

Sumita I, Bergman M (2009) Inner-core dynamics. In: Olson P (ed) Treatise on geophysics, vol. 8, core dynamics. Elsevier, Amsterdam, pp 299-318

Sumita I, Olson P (1999) A laboratory model for convection in Earth's core driven by a thermally heterogeneous mantle. Science 286:1547-9

Takemura S, Furumura T, Maeda T (2015) Scattering of high-frequency seismic waves caused by irregular surface topography and small-scale velocity inhomogeneity. Geophys J Int 201:459-74

Tanaka S, Hamaguchi H (1997) Degree one heterogeneity and hemispherical variation of anisotropy in the inner core from PKP(BC)-PKP(DF) times. J Geophys Res 102:2925-38

Tkalčić H (2015) Complex inner core of the Earth: the last frontier of global seismology. Rev. Geophys., 53: doi:10.1002/2014RG000469

Tkalčić H, Kennett BLN, Cormier VF (2009) On the inner-outer core density contrast from PKiKP/PCP amplitude ratios and uncertainties caused by seismic noise. Geophys J Int 179:425-43

Tkalčić H, Cormier VF, Kennett BLN, He K (2010) Steep reflections from the earth's core reveal small-scale heterogeneity in the upper mantle. Phys Earth Planet Inter 178:80-91
Tkalčić H, Chen Y, Liu R, Huang Z, Sun L, Chan W (2011) Multistep modelling of teleseismic receiver functions combined with constraints from seismic tomography: crustal structure beneath southeast China. Geophys J Int 187:303-26

Turcotte D, Schubert G (2002) Geodynamics, 2nd edn. Cambridge University Press, Cambridge

Waszek L, Irving J, Deuss A (2011) Reconciling the hemispherical structure of Earth's inner core with its super-rotation. Nat Geosci 4:264-7

Wessel P, Smith WHF (1998) New improved version of generic mapping tools released. EOS Trans Am Geophys Un 79:579

Yao J, Wen L (2014) Seismic structure and ultra-low velocity zones at the base of the Earth's mantle beneath Southeast Asia. Phys Earth Planet Inter 233: $103-11$

\section{Submit your manuscript to a SpringerOpen ${ }^{\circ}$ journal and benefit from:}

- Convenient online submission

- Rigorous peer review

- Immediate publication on acceptance

- Open access: articles freely available online

- High visibility within the field

- Retaining the copyright to your article

Submit your next manuscript at $\boldsymbol{s p r i n g e r o p e n . c o m ~}$ 